\title{
Measurement of Water Content in Solid Media by Means of Porous Ceramic Sensors
}

\author{
Hikoji WAKOH*, Toshisuke HIRANO ${ }^{\dagger}$
}

Received July 23, 1990; Accepted October 15, 1990

\begin{abstract}
A method for predicting transient one-dimensional (vertical) profiles of water content in solid media (substrates) has been developed. The transient water content was inferred on the basis of the relation between the relative water content in the substrate and the water matric potentials, which were measured continuously using porous ceramic sensors. This technique is of great advantage for nondestructive and simplified monitoring. For water content, the result is compared with the data reported by Timusk and Tenende. The water potentials are measured for typical artificial substrates. This method can be successfully monitored by applying the water potentials obtained using porous ceramic sensors to the newly derived relation.
\end{abstract}

\section{INTRODUCTION}

In modern horticulture, large quantities of artificial solid media (substrates) like rock wool instead of soil contribute to the cultivation at a number of plant factories and greenhouses. The fundamental functions of these substrates are to keep the water content suitable to successfully grow plants without interference with the transfer of water within the interstices of the substrates. Some of these substrates could be characterized by the abrupt change from water excess phenomenon to water deficient phenomenon. These phenomena deny crops. Since it is very difficult to observe these phenomena from the outside of the substrates, it is important to develop a monitoring method that is applicable for the measurement from the outside of the substrates to determine the distribution of the volumetric water content, $\theta$, on site and its

Industrial Research Institute of Kanagawa Prefecture, 3173 Showa-machi, Kanazawa-ku, Yokohama, 236, Japan

$\dagger$ Department of Reaction Chemistry, University of Tokyo, 7-3-1, Hongo, Bunkyo-ku, Tokyo, 113, Japan

Key Words: Water Management, Water Matric Potential, Porous Ceramic Sensor relative variation 1,2 ). Investigating the monitoring method will make it possible to develop optimum management schemes for water control with the purpose of obtaining the expected crops.

For the prediction, in general, of the distribution of $\theta$ and its relative variation, the water content is assumed to be at a static equilibrium as a function of depth and depends on the geometry of a substrate and on the wettability of the material. In the processes to determine these values, the effects of the roots involved and of the climatic environment have been disregarded 3,4 ). For the proper controlling of $\theta$ in an artificial substrate, the requirements that must be considered simultaneously for a practical method are as follows: (1) nondestructive, (2) continuous, (3) simple, and (4) employed for predicting at least a $\theta$-depth relation in the substrate during cultivation 5). To predict $\theta$ a considerable number of mathematical analyses and numerical simulations on $\theta$ have been performed on the basis of a diffusion equation. However, it seems extremely complicated to clarify the comparision of the predicted $\theta$ with the experimentally measured one 6,7 ). In practice, a 
drying method (gravimetric analysis) is unfit to continuously measure $\theta$ because a sample substrate must be destroyed for each measurement. Thus, the monitoring method for the necessary conditions of requirements (1) to (4) has not yet been established 8 10).

Most artificial substrates might specify a uniform structure and stable wettability, showing more uniform size and shape of the cross sections of a whole capillary tube than soil. The interstitial water exists mostly as capillary water 11). The amount of capillary water is related to the chemical potential, which is close to the magnitude of the matric potential, $\psi$, (negative pressure) ${ }^{12)}$. Recently, the continuous measurement of $\Psi$ has become simplified by using a relatively simple apparatus consisting of a tensiometer (porous cup), porous electrodes (ceramic sensor) and so on. Although in the previous studies on $\theta$, its prediction, based on the measured values of $\psi$, has been considered significant, the relationship between $\theta$ and $\psi$ and the construction of a reliable apparatus for measuring $\psi$ are essential to establish the monitoring method.

The present work describes a theoretical consideration of one-dimensional $\theta$ and a simplified method for monitoring $\theta$ in artificial substrates that does not take into account the plant roots and climatic environment. An equation to predict the distribution of $\theta$ is derived from a combination of the well-known Darcy law and the continuity equation provided that $\theta$ is governed by $\psi$ and that the sizes and shapes of the capillary tubes are in a stable and homogeneous form under a static equilibrium. The calculated distribution of $\theta$ is compared with the measured one reported by Timusk and Tenende 18). Typical calculated distributions are presented to show the availability of the ceramic sensor as a means of measuring $\varphi$.

\section{THEORY}

\subsection{Water content}

Water content, $\omega$, is defined Eq. (1): $\omega=\theta \cdot \varepsilon$ where $\theta$ is volumetric water content and $\varepsilon$ is porosity of the substrate. In dealing with $\theta$, one has usually assumed that $\theta$ depends on the matric potential, $\psi$, and the height, $z$, from a water table $(z=0)$. Under a static equilibrium, the value of $\theta$ must be 1 or zero corresponding to the height in which capillary water concentration is a maximum $(z=0)$ or zero ( $z=z \max$ ), respectively. It is generally assumed that $\psi$ depends on the size of the capillary tubes and their distribution. The size and the distribution account for individual certainty so that $\theta$ can be treated as a function of $\psi$ at each height. Hence, it is convenient to derive the relationship between $\theta$ and $\psi$ in advance for predicting the value of $\theta$ using the measured value of $\varphi$.

\subsection{Assumptions}

To simplify the procedure to predict $\theta$, the assumptions used are as follows:

(1) Both the amount, B, of water flowing in the substrate and the amount, $S$, of water absorbed by the roots are zero.

(2) $\psi$ is in a static equilibrium at a onedimensional coordinate (vertical) under the given conditions.

$$
\partial \psi / \partial t=0 \ldots \ldots \ldots \ldots \ldots \ldots \ldots \ldots
$$

where $t$ is time.

(3) The functional relationship between $\theta$ and $\psi$ is given by Eq. (3):

$$
\begin{aligned}
& \theta=1-\left(\Psi / \Psi_{\max }\right) \\
& =1-\Psi, \ldots \ldots .
\end{aligned}
$$

where $\psi \max$ is the maximum value of $\psi$ under various conditions and an empirical constant characterizing the individual substrate and $\Psi$ is the dimensionless potential.

(4) The hydraulic conductivity, $K$, is inversely proportional to $\Psi$ 13),

$$
K=-\mathrm{A} / \Psi \text {, (5) }
$$

where $A$ is a constant.

(5) The value of $\Psi$ is zero or 1 at $z=0$ or $Z=z_{\max }$, respectively, i.e.,

$$
\Psi=\left\{\begin{array}{l}
0 \text { at } z=0 \\
1 \text { at } Z=z_{\max } \ldots \ldots \ldots \ldots \ldots
\end{array}\right.
$$

The flow velocity of the osmotic water is less than the rate of $\mathrm{mm} /$ day. This fact suggests that 
water can be assumed as not going in and out of the substrate for several days, i.e., assumption (1) is reasonable. Many previous studies have been directed toward the measurement of the relationship between $\psi$ and $\theta$ or $K$ in different grain sizes of soil 14,15). It was found that $\theta$ was approximately linear with $\psi$ while $K$ was also approximately inversely proportional to $\psi$ in a range of $\psi$ though these relations are of somewhat defective reproducibility 16,17 ). The grain sizes in soil vary widely and form complicated internal structures which warp due to water absorption itself 14). The randomness of the size distributions might result in a lack of reproducibility. However, most artificial substrates have more homogenized geometry and wettability than soil. Their structures and other physical factors may be slightly altered by water absorption. There must be little effect of warp on $\psi$ in a homogenized substrate so that assumptions (3) and (4) hold for the water absorption.

\subsection{Distribution equation of $\theta$}

The one-dimensional transient distribution of $\theta$ in a stable and homogeneous soil is derived by the Richards' potential equation which is based on a combination of the well-known Darcy law and continuity equations under isothermal conditions 16 ).

$$
\begin{aligned}
& \frac{1}{\mathrm{C}(\Psi)} \frac{\partial}{\partial z}\left(K(\Psi)\left(\frac{\partial \Psi}{\partial z}+1\right)\right) \\
& -\frac{S}{\mathrm{C}(\Psi)}+\frac{B}{\mathrm{C}(\Psi)}=\frac{\partial \Psi}{\partial t}, \ldots
\end{aligned}
$$

where $\mathrm{C}$ is the specific water content and is -1 (the assumption (3)). Substituting Eqs. (2), (4) and (5) into Eq. (7), the following equation is given under assumption (1).

$$
\left.\Psi=\{\exp (z \cdot P)-1)\} / \exp \left(z_{\max } \cdot P\right)-1\right\}, \ldots
$$

where $P$ is the distribution function of $\psi$.

From Eqs. (4), (6) and (7), the following equation for $\theta$ is derived:

$$
\begin{aligned}
& \theta=\left\{\exp \left(z_{\max } \cdot P\right)-\exp (z \cdot P)\right\} \\
& \left\{\exp \left(z_{\max } \cdot P\right)-1\right\} . \ldots \ldots \ldots
\end{aligned}
$$

Equation (9) shows that the distribution of $\theta$ is expressed by an exponential function of $z$ ).

\subsection{Variation of $\theta$}

When $\psi$ varies from $\psi_{1}$ to $\psi_{2}$ at $z \quad(0<z<$ $z_{\text {max }}$ ), the difference of $\theta$ can be predicted by the following equation derived from Eq. (3):

$$
\theta_{2}-\theta_{1}=\left(\psi_{1}-\psi_{2}\right) / \psi_{\max } \text {. }
$$

When $z_{\max }$ changes from $z_{\max 1}$ to $z_{\max 2 \text {, the }}$ difference can be predicted by the following equation derived from Eq. (8):

$$
\begin{aligned}
& z_{\max 2}-z_{\max 1}=\frac{1}{P} l_{\mathrm{n}} \\
& \left\{\frac{\Psi_{1}}{\Psi_{2}}\left(\exp \left(z_{\max 1}-P\right)+1\right)-1\right\}-z_{\max 1}
\end{aligned}
$$

To predict $\theta$ and $z_{\max 2}$, we need the values of $\left(\varphi_{1}-\psi_{2}\right) / \psi_{\max }, \psi_{1} / \psi_{2}, P$ and $z_{\max 1}$. The values of $\left(\psi_{1}-\psi_{2}\right) / \psi_{\max }$ and $\psi_{1} / \psi_{2}$ can be measured with a porous ceramic sensor even if they vary during cultivation.

The value of $P$ can be predicted in the following way. If the substrate consists of only capillary tubes, then the value of $\psi_{\max }$ becomes,

$$
\psi_{\max }=\left[(2 \pi r) /\left(\pi r^{2}\right)\right] \cdot[(o \cos \alpha) /(\rho g)], \ldots
$$
where $r, a, \rho$ and $o$ are the capillary radius, contact angle, liquid density, and surface tension, respectively 12). In Eq. (12), [2nro $\cos a$ ] is related to the wet area and $\left[\pi \mathrm{r}^{2}\right]$ is related to the distance between pores in the substrate. The wet area and the distance depend on the packing density, $v$, of the substrate. The wet area is proportional to $v$, raised to the 1.5 power. The distance is inversely proportional to the third power of $v$. Thus, the relation between $P$ and $v$ becomes:

$$
P=E \cdot v \cdot 1.5 / z_{\max }
$$

where $E$ is a constant which is independent of $v$.

The value of $\theta$ for a given value of $v$ is derived from Eqs. (9) and (13), i.e.,

$$
\theta=\left\{\exp (E \cdot v-1.5)-\exp \left(E \cdot v-1.5 \cdot z / z_{\max }\right)\right] /\{\exp
$$

$(E \cdot v-1.5)-1\}$.

It is desirable to have calculations at different $v^{\prime}$ s for evaluating the value of $E$.

Basically, $z_{\max }$ is equal to $\psi_{\max }$. Substitution of this value into Eq. (3) and $z_{\max }$ becomes: 
Then, $\psi$ and $\theta$ at $z$ can be measured in advance of the cultivation with a porous ceramic sensor and gravimetric analysis by an apparatus like that illustrated in Fig. 1.

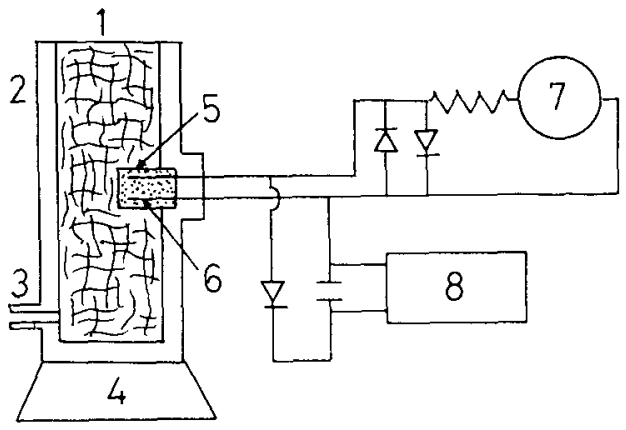

Fig. 1 Schematic diagram of apparatus for a continuous water potential measurement.

1. Substrate sample 2. Acrylic vessel wall 3. Spout

4. Electric balancer 5. Porous alumina sinterings 6 Au wire electrodes 7. Function generater $(100 \mathrm{~Hz}$, 2V) 8. Hybrid Recorder (DC $0-0.5 \mathrm{~V}, 30$ points)

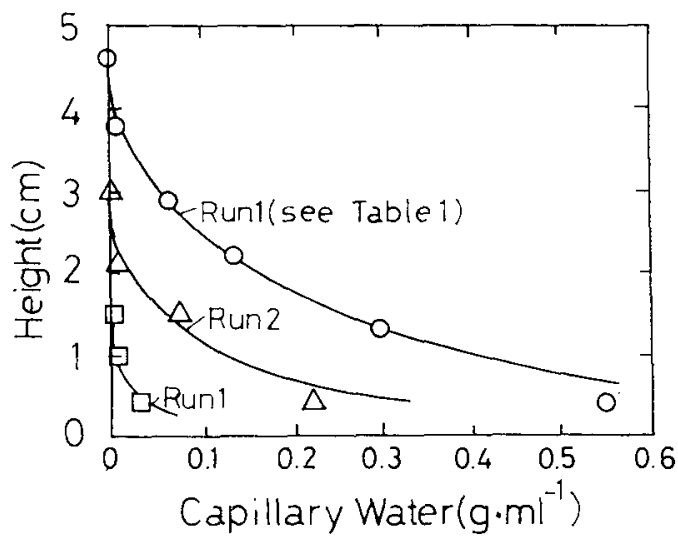

Fig. 2 Relation between the capillary water content and the height of capillary water above water table. Solid lines give the height where the water contents is $1 \%$ by volume as predicted by both Eq. (14) and the values shown in Table 1.

from their data. The values of $0.135 \mathrm{~g} \cdot \mathrm{ml}^{-1}, 0.100$ and 0.061 for $v$, and -0.2 for $E$ were adapted by fitting Eq. (14) to their data.

Table 1 Values used in Eq. (14) for the comparison the predicted and measured water content ${ }^{18)}$ presented in Fig. 2.

\begin{tabular}{|c|c|c|c|c|c|c|}
\hline \multirow[t]{2}{*}{ Run } & \multirow[t]{2}{*}{ Symbol } & \multicolumn{2}{|c|}{ Packing Density } & \multicolumn{2}{|c|}{ Constant } & \multirow{2}{*}{$\begin{array}{l}\text { Height b) } \\
Z_{\mathrm{max}} / \mathrm{cm}\end{array}$} \\
\hline & & $v / \mathrm{g} \cdot \mathrm{ml}^{-1}$ & $\left(v / g \cdot m l^{-1}\right)^{-1.5}$ & $\left(\mathrm{E} / \mathrm{g} \cdot \mathrm{ml}^{-1}\right)^{1.5}$ & $\mathrm{P} / \mathrm{cm}^{-1}$ & \\
\hline 1. & 0 & 0.135 & 20.2 & -0.2 & 0.88 & -4.6 \\
\hline 2. & $\triangle$ & 0.100 & 31.6 & -0.2 & 2.11 & -3.0 \\
\hline 3. & $\square$ & 0.061 & 66.4 & -0.2 & 8.85 & -1.5 \\
\hline
\end{tabular}

a) The density of the glass fibre board was increased through clamping.

b) The height of capillary water was distances from the free water surface.

\section{EXPERIMENTAL}

\subsection{Comparison with a gravimetric analysis}

The distribution of $\theta$ derived from Eq. (14) must be compared with that presented in previous studies before the reliability of the present measuring method is tested. The gravimetric analysis reported by Timusk and Tenende provides a good opportunity for such a comparison 18). They presented a water content that was determined gravimetrically for the water in glass fiber insulation in the frozen state after sufficiently infiltrating water into it. For this comparison the values of $v$ and $E$ were taken
A comparison of the results predicted using Eq. (14) with those by Timusk and Tenende 18) is presented in Fig. 2. The prediction was performed using the values presented in Table 1. In Fig. 2 an excellent agreement is seen between the predicted and measured water content profiles for different packing densities. These distribution curves are similar to the results that Taylor and Klepper found 1,19). Thus Eq. (9), giving $\theta$ as an exponential function of height, can be used for predicting water content for different packing densities of artificial substrates.

3.2 Measurement of water potential using porous ceramic sensors 
Table 2 Substrate samples and data of the retention water (bulk $1 \mathrm{~L}$ ).

\begin{tabular}{|l|c|c|c|c|c|}
\hline \multicolumn{1}{|c|}{ Run Specimen } & $\begin{array}{c}\text { Material } \\
\text { Artificial/ } \\
\text { Natural }\end{array}$ & $\begin{array}{c}\text { Packing } \\
\text { density } \\
v / \mathrm{g} \cdot \mathrm{ml}^{-1}\end{array}$ & Porosity & $\begin{array}{c}\text { Retention } \\
\text { water } \\
\omega / \mathrm{g} \cdot \mathrm{ml}^{-1}\end{array}$ & $\begin{array}{c}\text { Water } \\
\text { content } \\
\theta\end{array}$ \\
\hline 1. Rock wool (Nichi Asu) & Art. & 0.29 & 0.92 & 0.90 & 0.98 \\
2. $\quad$ Polyester (Germ, OD 30 $\mu \mathrm{m}$ ) & Art. & 0.10 & 0.92 & 0.78 & 0.81 \\
3. $\quad$ Peat moss (Canada, Heveco) & Nat. & 0.24 & 0.55 & 0.55 & 0.99 \\
4. $\quad$ Coconut husk (Bellabon 5M) & Nat. & 0.19 & 0.69 & 0.42 & 0.61 \\
5. Bark (Cryptomeria) & Nat. & 0.91 & 0.50 & 0.31 & 0.61 \\
6. Rock wool (Sin Nittetu) & Art. & 0.25 & 0.84 & 0.69 & 0.81 \\
7. Bark (Radiatus pine) & Nat. & 0.39 & 0.45 & 0.36 & 0.81 \\
8. Sand (Toyoura, OD 0.55 mm) & Nat. & 1.433 & 0.50 & 0.20 & 0.41 \\
9. Bark (Hemlock spruce) & Nat. & 0.56 & 0.48 & 0.22 & 0.46 \\
\hline
\end{tabular}

\subsubsection{Measurement of water potential As} mentioned previously the value of $\left(\psi_{1}-\psi_{2}\right) / \psi_{\max }$, $\psi_{1} / \psi_{2}$ and $\psi_{\max }\left(=z_{\max }\right)$ are for predicting water content variation and retention water, and are related with the magnitude of retention and repentance forces acting on the water excess or deficit phenomenon, the concentration gradient of water and solutes, and the movement of bubbles.

These values can be obtained directly either using a tensiometer or a porous ceramic sensor. Unfortunately, the first apparatus has trouble in that the pure water must be held inside the porous cup while it was being monitored. The second apparatus has the advantage of an impedance decrease which takes place as the sensor absorbs water. This apparatus may be maintenance free, so that it is suitable for continuous measurements. However, few results obtained using this sensor are available for the prediction of $\theta$ in artificial substrates 20 ).

3.2.2 Materials and methods The apparatus is schematically presented in Fig. 1. The sensor consists of a porous sintered alumina (Fujiwara Seisakusho, Tokyo. Outer diameter $0.6 \mathrm{~cm}$, length $0.6 \mathrm{~cm}$ ) containing two gold wires as electrodes. The relation between the pore size, $r$ in $\mathrm{cm}$, of the sintering and the osmotic pressure, $h$ in $\mathrm{cm}$, is given as $\mathrm{h} \sim 0.15 / \mathrm{r}$, which is derived from Eq. (12) assuming capillaries of equal radii 6 ). Sensors with ten different specifics $[p F$ values from 1.5 to 3.3 , interval 0.2 , where $p F$ is defined as $\log (\varphi / \mathrm{cm})]$ are installed on the wall of a cylindrical acrylic vessel (inner diameter $6 \mathrm{~cm}$, height $35.4 \mathrm{~cm}$ ) set on a balance. It is pierced with a small opening for the inlet and outlet in the wall at the height of $3 \mathrm{~mm}$ from the bottom.

Experiments were carried out on nine substrates shown in Table 2. Among them, the rockwool and polyester fiber are artificial materials. The substrates commercially available were used for tests without further treatment. After putting each sample in the vessel, water was injected through the opening into the vessel until it was filled. The impedance (at $100 \mathrm{~Hz}$ ) between the electrodes decreased when water infiltrated into the pores of the sintered alumina. Water drained spontaneously through the opening out of the vessel 30 minutes later. The changes in vessel weight and in the impedance were recorded continuously during the testing 21 ). 


\section{RESULTS AND DISCUSSION}

Results of the measurement of retention water content and $p F$ after an hour of drainage are presented in Table 2 and Fig. 3 . These

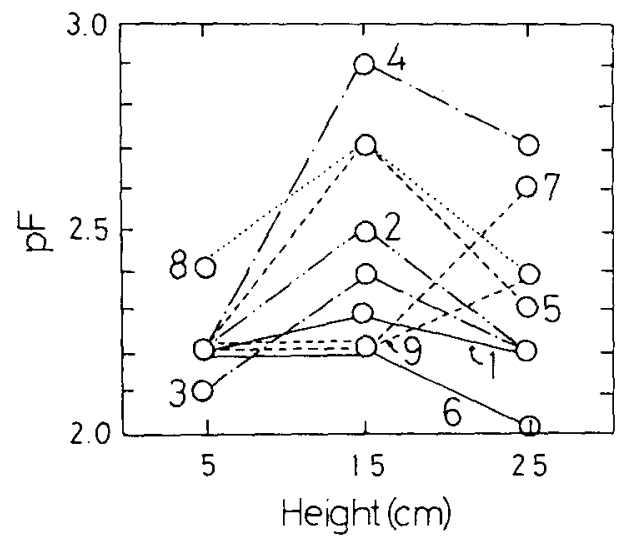

Fig. 3 Water potential $[p F=\log (\psi / \mathrm{cm})]$ curves for nine substrates measured using the porous electrodes. Numbers on lines denote the substrate specimens shown in Table 2. The height is above water table.

measured values have increased 5 to $10 \%$ two weeks later, indicating a rise in $p F$ corresponding to a slight evaporation of water. The observed values of $p F$ after an hour of drainage varied somewhat each time when samples were put in the vessel. These values were in an uncertain range of 0.2 to $0.5 \mathrm{pF}$.

There is a presumption that the values of $p F$ expected from Eq. (8) will increase in proportion to $z$ on logarithric scale. In practice, however, the expected values substantially deviate from the observed values. This is because the measured values include the intrinsic matric potential, the potential caused by an uneven packing, and the potential due to a loose contacting on the surface between the sample and sensor. It is difficult to perform a uniform packing and compact contacting for which the observed values are likely to give in the dispersal. Let us now estimate the effect of the extrinsic potentials on the deviations based on the average $M$ of the $p F$ values in Fig. 3 as well as the difference, $\Delta$, between the minimum $p F$ and the maximum $p F$. The maximum of $\Delta / M$ is 0.3 (sample 4 , Coconut husk, $M=2.6, \Delta=0.7$ ). The minimum of $\Delta / M$ is 0.05 (sample 1 , Rock wool, $M=2.2, \Delta=0.1$ ). If $M$ is less than $2.3, \Delta$ is less than 0.2 . These data suggest that the deviations, resulting from the loose contacting and uneven packing, are within 10 to $20 \%$ and about 10 to $20 \%$, respectively. The $\mathrm{pF}$ of 10 to $20 \%$ corresponds to the variation of 1.7 to 3.3 times $\theta$. It is certain that the extrinsic potentials associated with the loose contacting can not be directly distinguished from the observed values of $p F$. The absolute values obtained using this sensor are affected by the contacting situation.

In case the contacting situation holds constant throughout the monitoring, it is expected that the relative values of $\left(\psi_{1}-\psi_{2}\right) / \psi_{\max }$ and $\psi_{1} / \psi_{2}$ are compensated. These relative values are incorporated with Eq. (10) and we can get a clue to the continuous prediction of water content.

The water excess or deficit phenomenon may take place in bulk substrates when $p F_{\text {max }}$ becomes large and $\Delta$, in addition, becomes small. For each substrate, the calculated $p F_{\max }$ and the measured $\Delta$ are shown in Fig. 4 . The values of

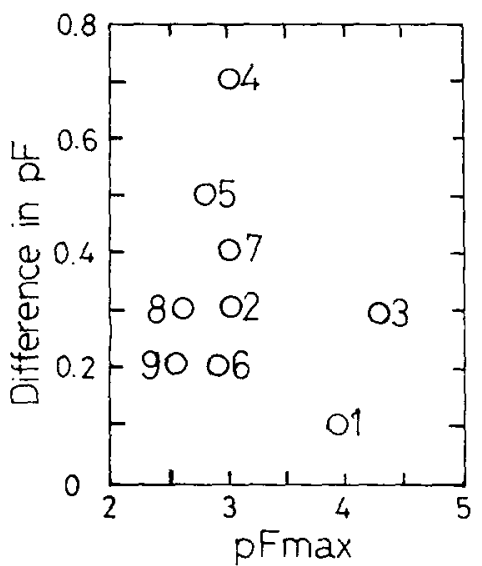

Fig. 4 The deviations $\Delta$ of water potential within individual substrate plotted against the $p F_{\max }$ (log $\left.z_{\text {max }}\right)$. The deviation is the difference between the maximum and the minimum $p F$ value shown in Fig. 3. The $p F_{\max }$ is calculated from Eq. (14). Each number indicates the substrate specimens shown in Table 2.

$p F_{\max }$ are calculated by substituting the measured values of $\psi$ presented in Fig. 3 and $\theta$ obtained from gravimetric data in Table 2 into Eq. (14). Figure 4 shows that the rock wool 
substrate (sample 1) has a large $p F_{\max }$ value of 3.9 and may have an apprehension of these phenomena. The polyester fiber substrate (sample 2) have a $p F_{\max }$ value of 3.0 as near natural substrate. The relative $p F$ values for artificial substrates may end up being used for advance estimating of its water content - matric potential relation.

\section{CONCLUSIONS}

The results of this study indicate that the monitoring method combined the relationship between water content and matric potential with porous ceramic sensors is suitable to predict water contents in artificial substrates. The relation for predicting transient one-dimensional (vertical) distribution of water content was derived, provided that the water content was governed by the matric potential and that the temporary variation of the matric potential was small. The results show an exponential increase in water content accompanying an arithmetic decrease in height under a static equilibrium. An agreement of the calculated values with the examined values concerning water contents suggests that this monitoring method has great potential for continuous managements where changes in water content within artificial substrates are desired. With future refinements in the compensation of extrinsic potentials which occur near the contact surface, porous ceramic sensors may be available for the prediction of water content in three-dimensional detail.

Contribution from the Horticultural Research Project, Kanagawa Prefecture.

\section{REFERENCES}

1) H. M. Taylor and B. Klepper, Soil Sci., 120, 57 67 (1975).

2) R. D. Bliesner, R. J. Hanks, L. G. King and L. S. Willardson, Soil Sci. Am.J., 41, 424-428 (1977).

3) D. Hillel, C. G. E. Beek and H. Talpaz, Soil Sci., 120,385-399(1975).

4) R. Steele, Textile Research J., 1958 Feb., 144147.

5) T. Sekiyama and K. Shirai, J.Agr.Met. (Japan), 41,131-137(1985).

6) D. Kirkham and W. L. Powers (eds.), Advanced Soil Physics, Wiley-Interscience, New York, p.320-342. (1972).

7) D. Hillel, H. Talpaz and H. V. Keulen, Soil Sci., 121, 242-255(1976).

8) J. K. M. Grinaven, H. W. G. Booltink, C. Dirksen, N. Breemen, N. Bongers and N. Waringa, Soil Sci. Am.J., 52, 1215-1218(1988).

9) J. M. Brown, W. C. Fonteno, D. K. Caseel and G. A. Johnson, Soil Sci. Am. J., 51, 1121-1125 (1987).

10) I. Miyazaki, T. Kasubuchi and S. Hasegawa, Trans. JSIDRE. (Japan), 119, 29-37 (1985).

11) M. I. Harpstead, F. D. Hole and W. F. Bennett (eds.), Soil Science Simplified, Iowa State University Press/Ames, p.71-87(1988).

12) H. G. Jones (eds.), Plants and microclimate, Cambridge Univ. Press, Cambridge, p.60-84 (1986).

13) S. J. Yang and E. Jong, Agron. J., 64, 574-578 (1972).

14) K. H. Hartge (eds.), Einfuhrung in die Bodenphysik, F. Enke Verlag, Stuttgart, (1978). (Japanese version: T. Fukushi, Water distribution in soil and hydrostatics, Hakuyuhsya, Tokyo, p.116-140).

15) G. S. Campbell, Soil Sci., 117, 311-314 (1974).

16) R. A. Feddes, P. Kabat, P. J. T. Bakel, J. J. B. Bronswijk and J. Halbertsma, J. Hydrology, 100, 69-111 (1988).

17) R. D. Jackson, Soil Sci. Soc. Am. Pro., 36, 380382 (1972).

18) J. Timusk and L. M. Tenende, J. Thermal Insulation, 11-April, 231-241 (1988).

19) M. N. Mimah and R. J. Hanks, Soil Sci. Soc. Amer.Proc., 37, 1121-1125(1973).

20) K. Nakatsuka, Y. Ikeuchi and K. Ishii, Japanese J.Soil Sci. Plant Nutr., 61, 193-195 (1990).

21) H. Wakoh and T. Hirano, 59th Spring Annual Meeting Chem. Soc. Japan. Abstr. 1-898 (1990). 